Highlights

An anytime tree search algorithm for the 2018 ROADEF/EURO challenge glass cutting problem Luc Libralesso,Florian Fontan

- We present the algorithm that allowed us to win the final phase of the 2018 ROADEF/EURO challenge.

- We introduce a new generic anytime tree search algorithm called Memory Bounded $A^{*}$.

- We outline guide functions, a symmetry breaking strategy, and a pseudo-dominance rule.

- We show by a comprehensive study that each of these components contributes to the algorithm global performance.

- We describe another tree search algorithm fully based on the pseudo-dominance rule and dedicated to instances with strong precedence constraints, for which it quickly finds the best-known solutions 


\title{
An anytime tree search algorithm for the 2018 ROADEF/EURO challenge glass cutting problem
}

\author{
Luc Libralesso $^{a, b}$, Florian Fontan ${ }^{a, b}$ \\ ${ }^{a}$ Univ. Grenoble Alpes, CNRS, Grenoble INP, G-SCOP, 38000 Grenoble, France \\ ${ }^{b}$ Each author contributes equally to this work.
}

\section{ARTICLE INFO}

\section{Keywords:}

Anytime tree search

Cutting \& Packing

2018 ROADEF/EURO challenge

Memory Bounded A*

\begin{abstract}
A B S T R ACT
In this article, we present the anytime tree search algorithm we designed for the 2018 ROADEF/EURO challenge glass cutting problem proposed by the French company Saint-Gobain. The resulting program was ranked first among 64 participants. Its key components are: a new search algorithm called Memory Bounded $\mathrm{A}^{*}\left(\mathrm{MBA}^{*}\right)$ with guide functions, a symmetry breaking strategy, and a pseudodominance rule. We perform a comprehensive study of these components showing that each of them contributes to the algorithm global performances. In addition, we designed a second tree search algorithm fully based on the pseudo-dominance rule and dedicated to some of the challenge instances with strong precedence constraints. On these instances, it finds the best-known solutions very quickly.
\end{abstract}

\section{Introduction}

In automated planning and scheduling (AI planning) communities, resolution methods often involve exploring a search tree. These methods usually perform an advanced greedy procedure. They explore the search tree starting with the parts that are evaluated a priori most promising and continue the exploration (so long as computational time is provided). Such methods are called anytime tree searches because they can be stopped at any time and provide good solutions relatively to the allowed computation time. Thus, they share the same purpose as classical meta-heuristics, but are less common in Operations Research. Still, one called Beam Search has shown a relative popularity in the Cutting \& Packing literature (Akeb et al., 2009; Bennell and Song, 2010; Baldi et al., 2014). They usually perform little inference within each node, and thus can open millions of nodes per second.

On the other hand, branch \& bound algorithms are ubiquitous in Operations Research. Such methods are usually designed to prove optimality, thus relying on strong bound computations (such as Lagrangian relaxations) and advanced pruning rules (dominances, symmetries, etc.) to reduce the size of the search tree as much as possible. However, such refined computations often drastically reduce the number of nodes opened per second. Consequently, the quality of solutions obtained on larger instances can be harmed due to fewer nodes opened.

The constructive nature of both anytime tree search algorithms and branch $\&$ bounds suggests that it could be possible to incorporate their respective advantages in a common approach. Indeed, some branch \& bound components may be (relatively) inexpensive to compute while still greatly reducing the search space. Using them within an anytime tree search algorithm would allow getting the best of both methods. It would provide a constructive method that is designed to find good solutions fast while taking advantage of the

luc. libralesso@grenoble-inp.fr (L. Libralesso); dev@florian-fontan.fr (F. Fontan)

ORCID(s): search space reductions from branch \& bounds.

With this in mind, we decided to develop such an algorithm for the 2018 ROADEF/EURO challenge glass cutting problem. We may note that this is unusual as almost all top-ranked methods in previous editions of the challenge mainly rely on local search or mathematical programming techniques.

We propose an anytime tree search with some simple bounds, pseudo-dominance properties, and symmetry breaking rules. We introduce some new guidance strategy that allows the algorithm to perform significantly better than if it was guided by a bound as in classical branch \& bound methods.

The search strategy can be roughly described as follows. It is a restarting strategy that starts its first iteration by performing very aggressive heuristic prunings. At the second iteration, it performs less aggressive heuristic prunings, taking more time than the previous iteration, but finding better solutions. If the algorithm runs long enough, some iteration may perform no heuristic pruning, thus the method will be able to guarantee optimality. The resulting method obtained the best results compared to the other submitted approaches during the final phase. We named it Memory Bounded $A^{*}$ as it performs a series of $\mathrm{A}^{*}$ with heuristic prunings which guarantee no-more than a given amount of nodes active at the same time.

We also highlight a general methodology that can be applied to other complex problems (and with other tree search algorithms). Indeed, the method can be divided into two parts: the Branching Scheme, usually problem-specific, which is a definition of the implicit search tree (i.e. root node, how to generate children of a given node, lower bounds, dominance rules, etc.); and a strategy, usually generic, to explore the tree. This decomposition allows rapid prototyping of both search tree definitions and tree search algorithms as many generic parts can be reused within other algorithms. It also helps to draw insights about the contribution of each component to the resulting search algorithm. 
An anytime tree search for the 2018 ROADEF/EURO challenge

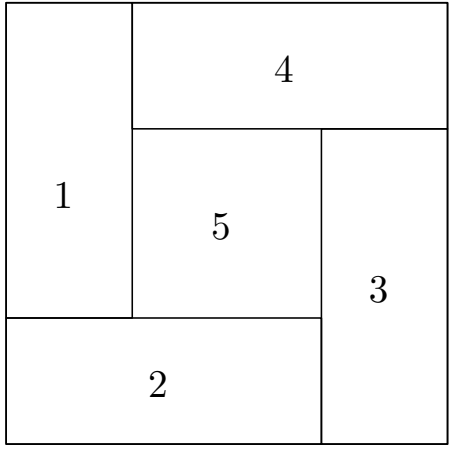

(a) Non-guillotine pattern

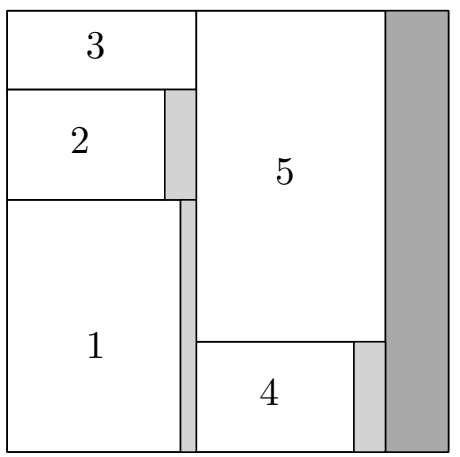

(b) Guillotine pattern

Figure 1: Illustration of a non-guillotine pattern (a) and a guillotine one $(b)$

This paper is structured as follows. In Section 2, we state the problem constraints and objective. In Section 3, we give some notations and definitions. In Section 4, we describe the branching scheme and in Section 5, the tree search algorithm we designed. Finally, in Section 6, we show the numerical results we obtained.

\section{Problem description}

The 2018 ROADEF/EURO challenge was dedicated to an industrial cutting problem from the French company SaintGobain. The challenge consists in packing rectangular glass items into standardized bins of dimensions $W \times H(6 \mathrm{~m} \times$ $3.21 \mathrm{~m})$.

The cutting plan needs to satisfy the following constraints:

- All items need to be produced

- Item rotation is allowed

- Cuts must be of guillotine type. Figure 1 illustrates two examples of non-guillotine and guillotine patterns. Furthermore, the number of stages is limited to four, with only one 4-cut allowed on a sub-plate obtained after 3-cuts. This configuration is close to classical three-staged non-exact guillotine patterns, but differs in that a sub-plate obtained after 3-cuts may contain two items as illustrated in Figure 2.

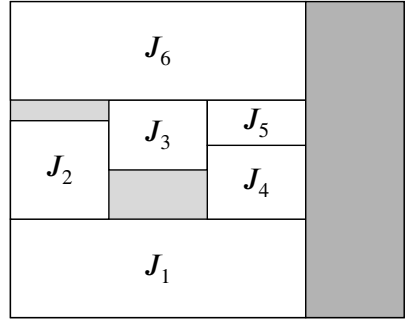

(a)

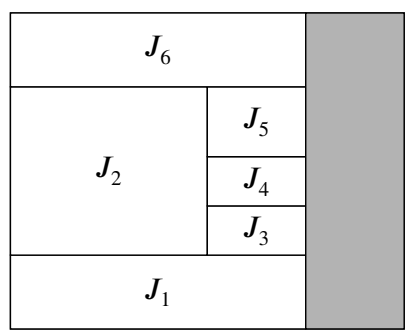

(b)

Figure 2: Only one 4-cut is allowed. Therefore, pattern (a) is feasible but pattern (b) is not

- Items are subject to chain precedence constraints. The extraction order is as follows: rightmost first level subplates first; within a first level sub-plate, bottommost second level sub-plates first; within a second level subplate, rightmost items first; and within a third level sub-plate, bottommost item first. Most instances have a dozen chains, three instances have 2 chains and five instances are not subject to precedence constraints.

- Bins contain defects (between 0 and 8 rectangles about a few centimeters high and wide). Items must be defectfree and it is forbidden to cut through a defect. Even if the bins have the same dimensions, the presence of defects makes the set of bins heterogenous. It is important to note that bins must be used in the order they are given.

- Depending on their level, sub-plates are subject to minimum and maximum size constraints. The width of first level sub-plates must lie between $w_{\min }^{1}=100$ and $w_{\max }^{1}=3500$, except for wastes. The height of second-level sub-plates must be at least $w_{\text {min }}^{2}=100$, except for wastes. Finally, the width and the height of any waste area must be at least $w_{\text {min }}=20$. This last constraint has an unusual consequence as illustrated in Figure 3.

The objective is to minimize the total waste area. It differs from classical Bin Packing Problems in that the remaining part of the last bin is not counted as waste. This objective is known in the packing literature as Bin Packing with Leftovers. It can be formulated as:

$\min n H W-H w-\sum_{i \in \mathcal{I}} w_{i} h_{i}$ 


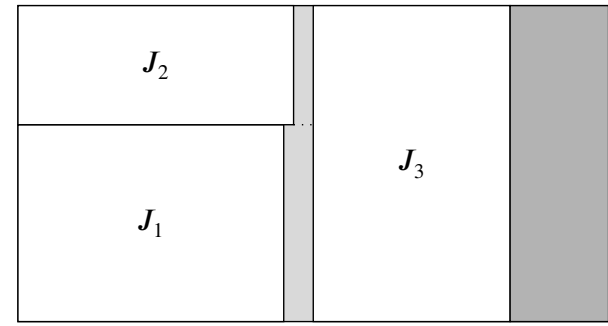

Figure 3: Optimal solution of the case containing the following three items with the chain precedence constraint $J_{1} \rightarrow J_{2} \rightarrow J_{3}$. Additional waste must be added before the first 1-cut. Otherwise either the waste area to the right of $J_{1}$ or the waste area to the right of $J_{2}$ would violate the minimum waste constraint.

where $n$ is the number of bins used; $W$ and $H$ are respectively the standardized width and height of the bins; $w$ is the position of the last 1-cut; $\mathcal{I}$ is the set of produced items; and $w_{i}$ and $h_{i}$ are respectively the width and the height of item $i \in \mathcal{I}$.

\section{Definitions and notations}

We use the following vocabulary: a $k$-cut is a cut performed in the $k$-th stage. Cuts separate bins or sub-plates in $k$-th level sub-plates. For example, 1 -cuts separate the bin in several first level sub-plates. $S$ denotes a solution or a node in the search tree.

We call the last first level sub-plate, the rightmost one containing an item; the last second level sub-plate, the topmost one containing an item in the last first level sub-plate; and the last third level sub-plate the rightmost one containing an item in the last second level sub-plate. $x_{1}^{\text {prev }}(S)$ and $x_{1}^{\text {curr }}(S)$ are the left and right coordinates of the last first level sub-plate; $y_{2}^{\text {prev }}(S)$ and $y_{2}^{\text {curr }}(S)$ are the bottom and top coordinates of the last second level sub-plate; and $x_{3}^{\text {prev }}(S)$ and $x_{3}^{\text {curr }}(S)$ are the left and right coordinates of the last third level sub-plate. Figure 4 presents a usage example of these definitions. We define the area and the waste of a solution $S$ as follows:

To compute area $(S)$ we distinguish two cases

- if $S$ contains all items:

$$
\operatorname{area}(S)=x_{1}^{\text {curr }}(S) h
$$

- and otherwise:

$$
\operatorname{area}(S)=\begin{aligned}
A & +x_{1}^{\text {prev }}(S) h \\
& +\left(x_{1}^{\text {curr }}(S)-x_{1}^{\text {prev }}(S)\right) y_{2}^{\text {prev }}(S) \\
& +\left(x_{3}^{\text {curr }}(S)-x_{1}^{\text {prev }}(S)\right)\left(y_{2}^{\text {curr }}(S)-y_{2}^{\text {prev }}(S)\right)
\end{aligned}
$$

We compute the waste of a partial solution as follows:

$$
\operatorname{waste}(S)=\operatorname{area}(S)-\text { item_area }(S)
$$

with $A$ the sum of the areas of all but the last bin, $h$ the height of the last bin and item_area $(S)$ the sum of the area of the items of $S$. Area and waste are illustrated in Figure 4.

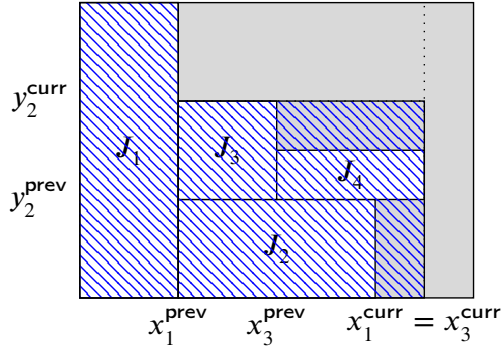

Figure 4: Last bin of a solution which does not contain all items. The area is the whole hatched part and the waste in the grey hatched part.

\section{Branching scheme}

\subsection{General scheme}

Two kinds of packing strategies are used in the packing literature: item-based and block-based. In item based strategies, only one item is inserted at each step, whereas in blockbased strategies, multiple items are inserted. Although several researchers highlighted the benefits of block-based approaches (Bortfeldt and Jungmann, 2012; Wei et al., 2014; Lodi et al., 2017), we chose an item-based strategy. Two reasons support this choice. First, the problem has more constraints than classical packing problems from the literature. Thus, generating feasible solutions is already challenging and block-based approaches add even more complexity. Second, the benefits of the block-based approaches might be compensated by a more powerful tree search algorithm.

However, our strategy is not purely item-based: instead of packing one item at each step, we pack the next third level sub-plate. This comes from the observation that because only one 4-cut is allowed in a third level sub-plate, a third level sub-plate has only five possible configurations; it may contain

1. exactly one item, without waste

2. exactly one item with some waste above

3. exactly one item with some waste below

4. exactly two items, without waste

5. no item, only waste

These configurations are illustrated in Figure 5. The subplates containing $J_{1}$ and $J_{2}$ respectively follow configurations 1 and 2. These are the "standard" configurations. Placing an item on top of the sub-plate as in configuration 3 may be necessary if it would contain a defect otherwise as $J_{3}$. Similarly, inserting only waste (configuration 5) may also be necessary if the region contains a defect as the sub-plate containing the second defect. We do not allow directly inserting only waste in a region containing no defects. Such sub-plate may appear in a solution, as the third-level sub-plate to the right of $J_{4}$ and $J_{5}$, but it is implicitly generated when $J_{6}$ is inserted. Finally, the sub-plate containing items $J_{4}$ and $J_{5}$ corresponds to configuration 4.

Third level sub-plates are inserted in the order they are extracted. In Figure 5, this follows the numbering of the 


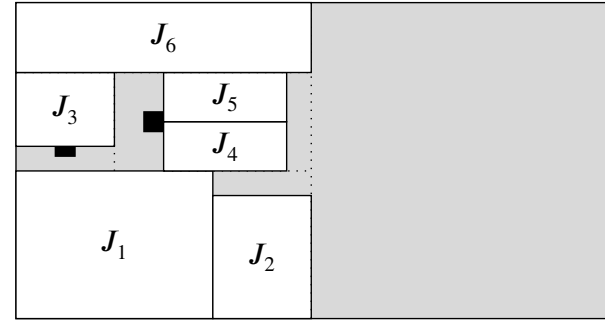

Figure 5: Illustration of third level sub-plate possible configurations. Black rectangles are defects.

items. This ensures to never violate the precedence constraints. All items are candidates if their insertion does not lead to a precedence constraint violation.

Then, a third level sub-plate can be inserted at several depths

- depth 0: in a new bin

- depth 1: in a new first level sub-plate to the right of the current one

- depth 2: in a new second-level sub-plate above the current one

- depth 3: in the current second-level sub-plate already, to the right of the last third-level sub-plate

To reduce the size of the tree, we apply some simple pruning rules:

- if a third-level sub-plate can be inserted in the current bin, we do not consider insertions in a new bin; and if a third level sub-plate can be inserted in the current first (resp. second) level sub-plate without increasing the position of its left 1-cut (resp. top 2-cut), we do not consider insertions in a new first (resp. second) level sub-plate;

- If the last insertion is an empty sub-plate at depth $d$, then the next insertion must also happen at depth $d$;

- If the last insertion is a 2-item insertion at depth $d \neq 3$, then the next insertion must be at depth 3 .

With this branching scheme, item rotation and minimum and maximum distances between cuts constraints are easy to take into account.

\subsection{Pseudo-dominance rule}

In this section, we describe a more sophisticated heuristic dominance rule. For a (partial) solution, we define its "front" as the polygonal chain

$$
\begin{aligned}
& \left(\left(x_{1}^{\text {curr }}, 0\right),\left(x_{1}^{\text {curr }}, y_{2}^{\text {prev }}\right),\left(x_{3}^{\text {curr }}, y_{2}^{\text {prev }}\right),\right. \\
& \left.\left(x_{3}^{\text {curr }}, y_{2}^{\text {curr }}\right),\left(x_{1}^{\text {curr }}, y_{2}^{\text {curr }}\right),\left(x_{1}^{\text {curr }}, h\right)\right)
\end{aligned}
$$

Figure 6 shows two examples of solution fronts.

Then we say that solution $S_{1}$ dominates solution $S_{2}$ iff they contain the same items and the front of $S_{1}$ is "before" the front of $S_{2}$. (see Figure 7).

If the number of possible subsets of items is small, then for a given subset, we can memorize the best front currently
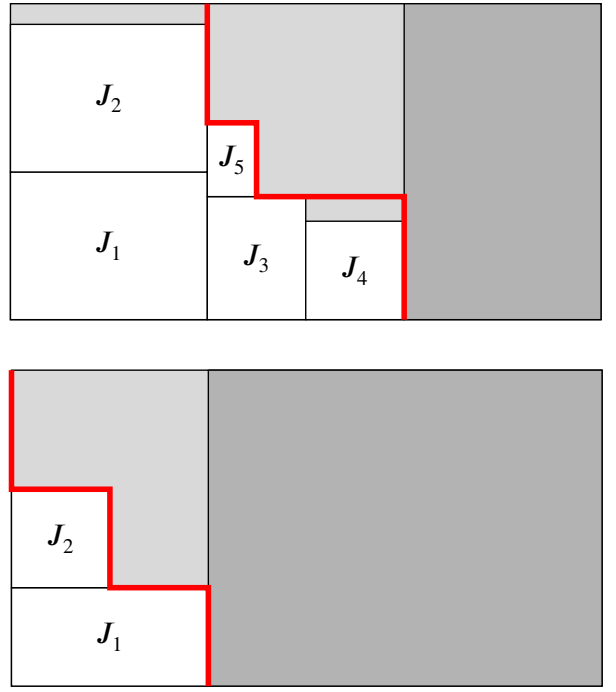

Figure 6: Illustration of the front of two partial solutions

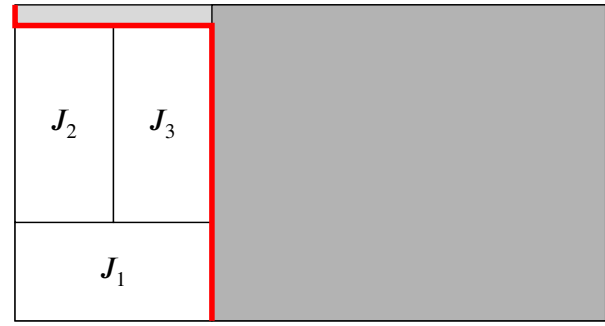

(a)

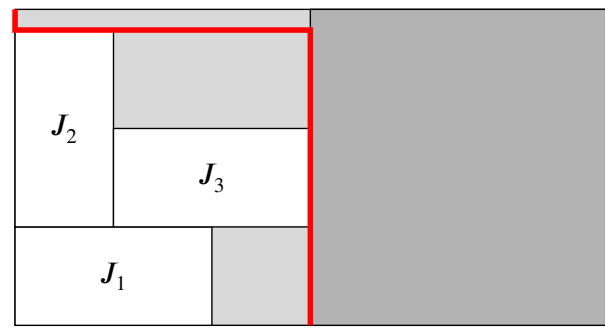

(b)

Figure 7: Solution (a) dominates solution (b)

seen during the search and prune any new dominated node encountered. This situation occurs in instances with strong precedence constraints (i.e. two chains) and this is the strategy of the DPA* algorithm presented afterward. However, for most instances, the number of possible subsets is too large and we only use the pseudo-dominance rule among the children of a node. To compensate, an additional symmetry breaking strategy is introduced.

\subsection{Symmetry breaking strategy}

We designed the following symmetry breaking strategy: if they do not contain defects and can be exchanged without violating the precedence constraints, a $k$-level sub-plate is forbidden to contain an item with a smaller index than the previous $k$ level sub-plate of the same $(k-1)$-level sub-plate. 


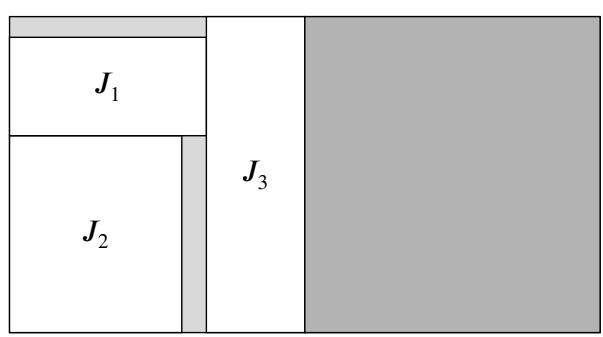

(a)

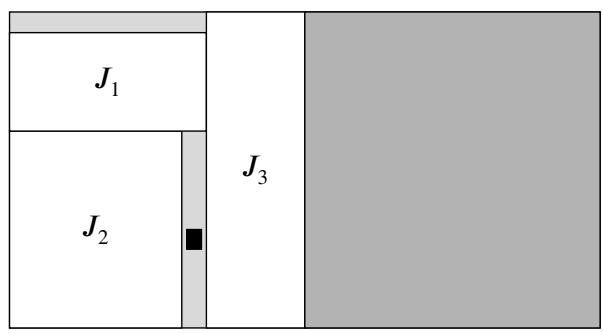

(b)

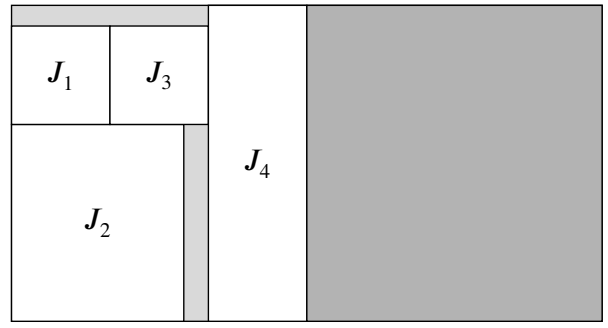

$J_{1}, J_{2} \rightarrow J_{3}$

(c)

Figure 8: Illustration of the symmetry breaking strategy: pattern $(a)$ is forbidden because the second-level sub-plates containing $J_{1}$ and $J_{2}$ can be exchanged without a feasibility issue. However, pattern (b) is allowed because of the defect and pattern (c) is also allowed because if the second-level sub-plates are exchanged, then the precedence constraint between $J_{2}$ and $J_{3}$ is violated.

Preliminary experiments showed that applying the strategy for $k=2$ and $k=3$ yield the best results. The symmetry breaking strategy is illustrated in Figure 8.

It should be noted that the branching scheme is not dominant, i.e. for some instances, it may not contain an optimal solution. Likewise, the pseudo-dominance rule considers that solution $S_{1}$ dominates solution $S_{2}$ whereas no optimal solution can be reached from $S_{1}$ but one can be from $S_{2}$. More details about this are given by Fontan (2019).

\section{Tree search}

During our initial work on the challenge, we first explored the classical "Operations Research" optimization algorithms (local-search, evolutionary algorithms and branch and bounds). However, it seemed difficult for us to find efficient localsearch or evolutionary moves, while it felt relatively natural to design constructive methods. We implemented sev- eral classical constructive algorithms: a greedy algorithm quickly providing solutions but with limited quality; a Best First (A*) algorithm returning the "optimal" one (relatively to the branching scheme) on small instances; and a Depth First struggling to improve the greedy solution.

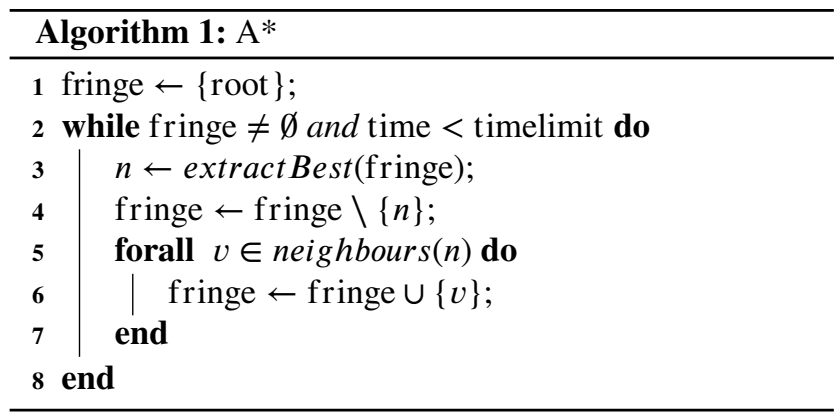

We remind the pseudo-code of the $\mathrm{A}^{*}$ algorithm in Algorithm 1. At each iteration, the "best" node is extracted from the fringe and its children are added to the fringe. As written above, our implementation of $A^{*}$ was able to find the optimal solutions on very small instances but was quickly running out of memory larger ones because of the size of the fringe. Therefore, we decided to heuristically prune nodes to bound the required memory. This "heuristic" algorithm performed beyond expectations and provided excellent solutions. However, it depended on the amount of memory allowed for the fringe. If this parameter is too small, the search ends quickly and not benefit from the remaining available time. If too big, the search takes more time and does not provide any solution within the time limit. To get rid of this parameter, we chose to use a restart strategy where we geometrically increase the allowed memory at each restart. The new parameter to calibrate becomes the growth factor, but we found that any value between 1.25 and 3 provided similar results. This simple approach provided good solutions.

Motivated by this simple but yet efficient algorithm, we investigated other anytime tree search algorithms such as beam search (Ow and Morton, 1988) and beam stack search (Zhou and Hansen, 2005). We implemented and compared them on the challenge problem. To our surprise, they did not perform as well as the previously described approach. To the best of our knowledge, this approach has not been used in the Operations Research literature before. We describe it in more detail in the next section.

\subsection{Memory Bounded $A *(M B A *)$}

A* is known to minimize the cost estimate on nodes it opens. However, it suffers from a large memory requirement since it has to store a large number of nodes in the fringe. We propose a simple but yet powerful heuristic variant of A* that cuts less promising nodes if the size of the fringe goes over a parameter $D$. We call this tree search algorithm Memory Bounded $A^{*}\left(M B A^{*}\right)$. If $D=1$, it generalizes a greedy algorithm and if $D=\infty$, it generalizes $A^{*}$.

The pseudo-code of MBA* is given in Algorithm 2. Only lines 8 to 11 are added compared to the $A^{*}$ algorithm pre- 
sented in Algorithm 1. MBA* starts with a fringe containing only the root node (line 1). At each iteration, the "best" node is extracted from the queue (lines 3 and 4) and its children are added to the queue (lines 5 to 7 ). If the size of the queue goes over $D$, the "worst" nodes are discarded (lines 8 to 11).

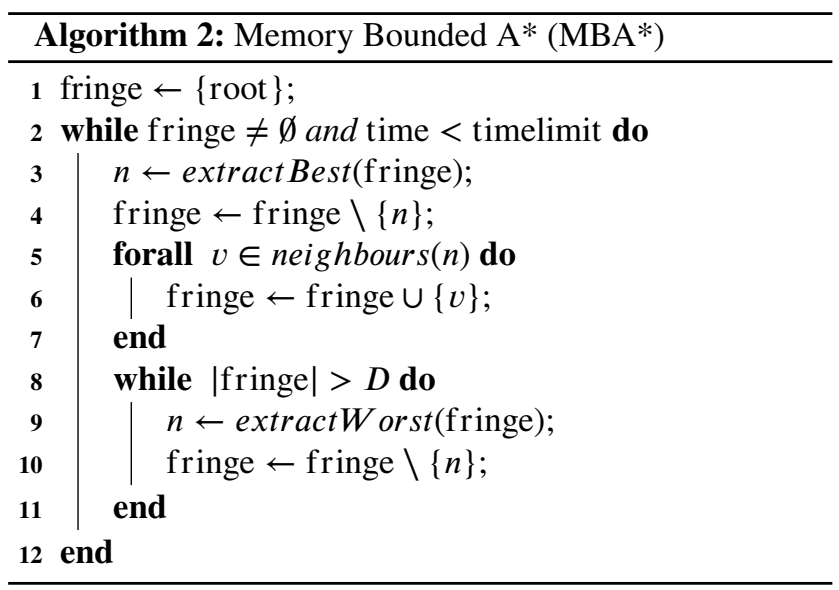

\subsection{Guide functions}

Tree search methods are dependent on well-crafted guide functions which define the meaning of "best" and "worst" nodes. Using a lower bound is common in the tree search literature. Indeed, if the objective is to prove optimality, using a lower bound as a guide function will minimize the number of opened nodes. Therefore, we first tried this approach and used the waste as a guide function. We noticed that the resulting solutions packed small items on the first plates and big items on the last ones, thus generating little waste in the beginning but a lot in the end. Globally, the solution quality was not satisfactory as illustrated in Figure 9a.

Taking this into account, we designed new guides to balance the cost of inserting small items at the beginning of the solutions:

\section{waste percentage (= waste / area):}

compared to waste only, the waste has less impact if the solution contains larger items.

waste percentage / average area of packed items: this guide function directly adds a reward to solutions containing large items.

The benefit of these guides is illustrated in Figure $9 \mathrm{~b}$.

\subsection{DPA*: solving instances with strong precedence constraints}

Three instances of the challenge contain only 2 precedence chains. If we denote by $n_{1}$ (resp. $n_{2}$ ) the length of the first (resp. second) chain, then the number of possible subsets of items packed in a partial solution of the branching scheme becomes $n_{1} n_{2}$. Since the number of items in an instance is less than 700 (this information was given in the challenge description), it becomes possible to store the nondominated fronts encountered for each possible subset without overcoming the memory limitation, compare the front of each opened node with the non-dominated fronts from all the previously encountered nodes and prune the dominated ones.

Therefore, we developed a dedicated algorithm for these instances named Dynamic Programming A* (DPA*). DPA* is an $\mathrm{A}^{*}$ algorithm implementing the scheme described in the previous paragraph. DPA* does not bound the size of the queue as MBA* does, and uses the waste as a guide function. Therefore, if it terminates, it returns the "optimal" solution (relatively to the branching scheme and the pseudodominance rule).

We may note that for a given subset of items, there could be an exponential number of non-dominated fronts, which could degrade DPA* performances. This is at least not an issue for the concerned instances from the challenge.

\subsection{Global algorithm}

For the competition, we distinguished the case where the instance has two chains or less. In this case, we run DPA*.

If it has strictly more than two chains, we do not use DPA* since it would overcome the memory limitation. Since the processor used to evaluate the participant submissions had 4 physical cores, we run 4 threads, each one running a restarting $\mathrm{MBA}^{*}$ with a given growth factor and a given guide function. Each MBA* is initially executed with a fringe maximal size of 2 , and each time one terminates, it is restarted with a maximal fringe size multiplied by its growth factor. If the growth factor is 2 , the maximal size doubles at each iteration. All the threads share the information of the best solution found. If one finds a better solution, the others take advantage of it to perform more cuts and globally perform better together than alone. The threads run the following algorithms:

- $\mathrm{MBA}^{*}$, waste percentage guide, growth factor 1.33

- $\mathrm{MBA}^{*}$, waste percentage guide, growth factor 1.5

- MBA*, waste percentage / average size guide, growth factor 1.33

- MBA*, waste percentage / average size guide, growth factor 1.5

\section{Numerical results}

In this section, we first evaluate the contribution of the components we described in the previous sections in the main algorithm. Then, we show the benefits of using DPA* on instances with only two precedence chains. Finally, we provide computational results with the challenge setting. Instances generally have between 300 and 600 items and 10 to 15 chains. They are available online ${ }^{1}$.

\subsection{Contribution of the components}

In this section, computational experiments have been performed on a personal computer with an Intel(R) Core(TM) i5-3470 CPU @ 3.20GHz with 16GB RAM.

\footnotetext{
$1_{\text {https: //www. roadef.org/challenge/2018/en/instances. php }}$
} 

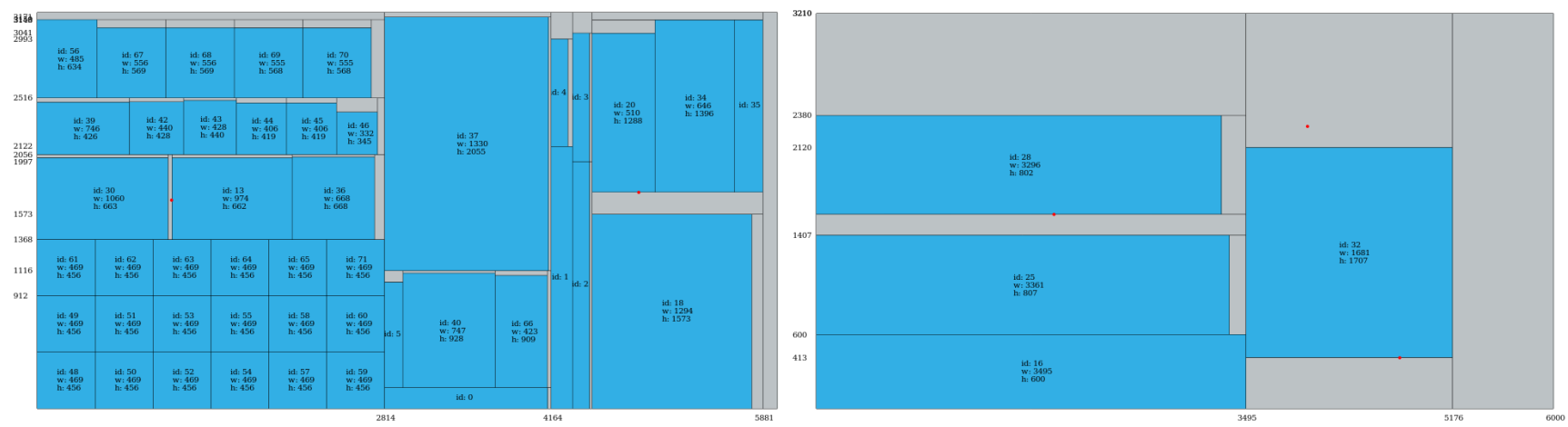

(a) waste only guide - first and last plate
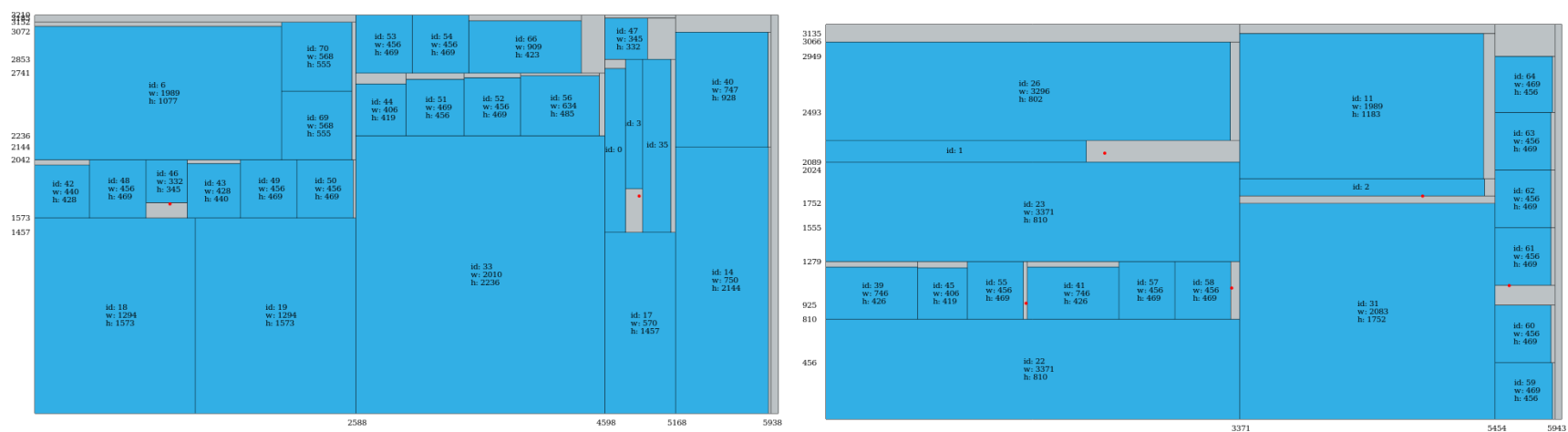

(b) waste / average size guide - first and last plate

Figure 9: 9a shows a solution obtained using the waste as guide function. Notice that at the beginning of the solution, small items are omnipresent whereas in later plates, only large items are present, thus globally generates more waste. $9 \mathrm{~b}$ shows the effect of the guide biased by the item average size on a solution of the same instance. We observe that small and large items are better mixed and significantly less waste is generated at the end of the solution.

We consider the 12 possible combinations of the components we designed, namely $\mathrm{MBA}^{*}$ to be compared with an Iterative Beam Search Zhang (1998); with or without the symmetry breaking strategy; and with waste (w), waste percentage (p), or waste percentage/average size (a) guide. We run each pair of instance-algorithm for 100 seconds.

In Table 1, we show a summary of the performances of each variant. The goal is to help us select the best combinations to use. MBA* with the symmetry breaking strategy and guided by the waste percentage $\left(M B A^{*}+s y m+p\right)$ and MBA* with the symmetry breaking strategy and guided by the waste percentage / average size $\left(M B A^{*}+s y m+a\right)$ clearly outperform all other combinations. That is why these are the two combinations that we use. Since the processor used to evaluate participant submissions has 4 physical cores, we dedicate two threads for each combination with different growth factor (1.33 and 1.5) to add some robustness.

Table 2 presents an analysis of the contribution of each component individually. Each column corresponds to the best result per instance obtained by a subset of algorithms that uses a given component. For instance best IBS corresponds to a subset of algorithms using Iterative Beam Search, thus excluding MBA* (6 algorithms). MBA* variants outperform the Iterative Beam Search variants (producing $12 \%$ less waste). It finds $41 / 50$ best solutions and 36 best so-

\begin{tabular}{|c|c|c|}
\hline Combination & \# best & \# only best \\
\hline BS+no-sym+w & 2 & 0 \\
\hline BS+no-sym+p & 2 & 0 \\
\hline BS+no-sym+a & 2 & 0 \\
\hline $\mathrm{BS}+\mathrm{sym}+\mathrm{w}$ & 5 & 1 \\
\hline BS+sym+p & 9 & 4 \\
\hline $\mathrm{BS}+\mathrm{sym}+\mathrm{a}$ & 9 & 4 \\
\hline $\mathrm{MBA}^{*}+$ no-sym $+\mathrm{w}$ & 2 & 0 \\
\hline $\mathrm{MBA}^{*}+$ no-sym $+\mathrm{p}$ & 3 & 0 \\
\hline $\mathrm{MBA}^{*}+$ no-sym $+\mathrm{a}$ & 4 & 0 \\
\hline $\mathrm{MBA}^{*}+\mathrm{sym}+\mathrm{w}$ & 3 & 0 \\
\hline $\mathrm{MBA}^{*}+\mathrm{sym}+\mathrm{p}$ & 23 & 17 \\
\hline $\mathrm{MBA}^{*}+\mathrm{sym}+\mathrm{a}$ & 22 & 18 \\
\hline
\end{tabular}

Table 1

Comparison over all possible algorithms using the proposed algorithmic components. "\# best" indicate the number of times the algorithm was able to find the best solution on given instances compared to the 11 other algorithms. "\# only best" indicates the number of times the algorithm was the only one to find the best solution.

lutions that the Beam Search variants were not able to obtain. The algorithms using the symmetry breaking strategy clearly produce better results than the one without (13\% less waste and 46 best solutions not attainable by the variants 


\begin{tabular}{|c|c|c|c|c|c|c|c|}
\hline Instance & best IBS & best MBA* & best no sym & best with sym & best $w$ & best $p$ & best a \\
\hline $\mathrm{A} 1$ & 425486 & 425486 & 425486 & 425486 & 425486 & 425486 & 425486 \\
\hline A2 & 10514609 & 9676799 & 10537079 & 9676799 & 10659059 & 10418309 & 9676799 \\
\hline A3 & 2651880 & 2651880 & 3441540 & 2651880 & 3056340 & 2651880 & 2651880 \\
\hline A4 & 3242520 & 3220050 & 3306720 & 3220050 & 3505740 & 3220050 & 3306720 \\
\hline A5 & 3033273 & 3566133 & 4856553 & 3033273 & 3736263 & 3033273 & 3566133 \\
\hline A6 & 3225930 & 3572610 & 3652860 & 3225930 & 3800520 & 3225930 & 3460260 \\
\hline A7 & 5063280 & 4800060 & 5194890 & 4800060 & 5933190 & 4800060 & 4938090 \\
\hline A8 & 9187874 & 10077044 & 12218114 & 9187874 & 12568004 & 10077044 & 9187874 \\
\hline A9 & 2930706 & 2985276 & 3550236 & 2930706 & 3929016 & 2930706 & 2985276 \\
\hline A10 & 4097221 & 4084381 & 5272081 & 4084381 & 4797001 & 4084381 & 4122901 \\
\hline A11 & 4718449 & 4978459 & 6076279 & 4718449 & 6711859 & 5251309 & 4718449 \\
\hline A12 & 2050084 & 2245894 & 2342194 & 2050084 & 2050084 & 2104654 & 2194534 \\
\hline A13 & 15096453 & 12133623 & 14865333 & 12133623 & 15099663 & 12197823 & 12133623 \\
\hline A14 & 14363778 & 12097518 & 14793918 & 12097518 & 14363778 & 12097518 & 13490658 \\
\hline A15 & 15277961 & 13185041 & 15168821 & 13185041 & 16029101 & 13185041 & 15014741 \\
\hline A16 & 3380333 & 3380333 & 3380333 & 3380333 & 3380333 & 3380333 & 3380333 \\
\hline A17 & 3617251 & 3617251 & 3617251 & 3617251 & 3617251 & 3617251 & 3617251 \\
\hline A18 & 5898468 & 5535738 & 5763648 & 5535738 & 7737798 & 5596728 & 5535738 \\
\hline A19 & 3323744 & 3654374 & 4187234 & 3323744 & 4620584 & 3323744 & 3965744 \\
\hline A20 & 1467925 & 1467925 & 1493605 & 1467925 & 1467925 & 1467925 & 1467925 \\
\hline B1 & 4173228 & 3633948 & 4150758 & 3633948 & 4012728 & 4324098 & 3633948 \\
\hline B2 & 15715685 & 15359375 & 18466655 & 15359375 & 20155115 & 15359375 & 15715685 \\
\hline B3 & 32668193 & 21253433 & 23365613 & 21253433 & 41315933 & 24890363 & 21253433 \\
\hline B4 & 8885365 & 8862895 & 11238295 & 8862895 & 9222415 & 8862895 & 8920675 \\
\hline B5 & 92433185 & 88590815 & 88590815 & 88590815 & 103719545 & 88590815 & 88590815 \\
\hline B6 & 13371637 & 13480777 & 15653947 & 13371637 & 17509327 & 14113147 & 13371637 \\
\hline B7 & 14576799 & 11434209 & 12801669 & 11434209 & 14319999 & 11434209 & 12801669 \\
\hline B8 & 24490999 & 19512289 & 24121849 & 19512289 & 24490999 & 19512289 & 20048359 \\
\hline B9 & 20511607 & $\begin{array}{lll}20 & 046157\end{array}$ & 25085857 & 20046157 & 46721257 & 39071827 & $\begin{array}{lll}20 & 046 & 157\end{array}$ \\
\hline B10 & 28012013 & 27344333 & 29225393 & 27344333 & 35815523 & 31055093 & 27344333 \\
\hline B11 & 38143250 & 29113520 & 34175690 & 29113520 & 41523380 & 32589950 & 29113520 \\
\hline B12 & 18122077 & 16086937 & 19929307 & 16086937 & 18122077 & 16086937 & 16314847 \\
\hline B13 & 31138545 & 29674785 & 33716175 & 29674785 & 31138545 & 32213895 & 29674785 \\
\hline B14 & 10482820 & 10043050 & 11619160 & 10043050 & 12046090 & 10434670 & 10043050 \\
\hline B15 & 41533241 & 28372241 & 34143821 & 28372241 & 41533241 & 28372241 & 31466681 \\
\hline $\mathrm{X} 1$ & 21022877 & $\begin{array}{ll}17299 & 277\end{array}$ & 17970167 & $\begin{array}{ll}17299 & 277\end{array}$ & 29911367 & 17803247 & $\begin{array}{lll}17 & 299 & 277\end{array}$ \\
\hline$\times 2$ & 11459837 & 8583677 & 8923937 & 8583677 & 9318767 & 9206417 & 8583677 \\
\hline X3 & 9424756 & 8712136 & 9842056 & 8712136 & 9578836 & 8712136 & 8927206 \\
\hline$\times 4$ & 19035422 & 15976292 & 19305062 & 15976292 & 19035422 & 15976292 & 16772372 \\
\hline$\times 5$ & 5383037 & 5620577 & 7029767 & 5383037 & 6728027 & 5623787 & 5383037 \\
\hline $\mathrm{X} 6$ & 14443523 & 12167633 & 14488463 & 12167633 & 14443523 & 13024703 & 12167633 \\
\hline $\mathrm{X} 7$ & 30327120 & 26170170 & 27146010 & 26170170 & 31328640 & 29161890 & 26170170 \\
\hline $\mathrm{X} 8$ & 27693711 & 27109491 & 27693711 & 27109491 & 27693711 & 27494691 & $27 \quad 109491$ \\
\hline$\times 9$ & 33431655 & 23599425 & 33919575 & 23599425 & 33370665 & 23599425 & 26716335 \\
\hline X10 & 23400722 & 19901822 & 23522702 & 19901822 & 23673572 & 23975312 & 19901822 \\
\hline X11 & 14349972 & 14247252 & 16102632 & 14247252 & 14349972 & 14247252 & 14921352 \\
\hline $\mathrm{X} 12$ & 14775805 & 12422875 & 14576785 & 12422875 & 14775805 & 12422875 & 12589795 \\
\hline $\mathrm{X} 13$ & 19208322 & 14624442 & 18900162 & 14624442 & 20007612 & 16271172 & 14624442 \\
\hline X14 & 11075552 & 9730562 & 11916572 & 9730562 & 11004932 & 9730562 & 10128602 \\
\hline X15 & 16301394 & 13540794 & 17261184 & 13540794 & 16461894 & 13990194 & 13540794 \\
\hline total waste & 779159574 & 679871064 & 779027964 & 676914654 & 870817914 & 725241204 & 693016014 \\
\hline $\mathrm{nb}$ best & 14 & 41 & 4 & 50 & 5 & 27 & 28 \\
\hline $\mathrm{nb}$ only best & 9 & 36 & 0 & 46 & 1 & 21 & 22 \\
\hline
\end{tabular}

Table 2

Analysis of the contribution of each introduced algorithmic component 
An anytime tree search for the 2018 ROADEF/EURO challenge

\begin{tabular}{l|c|c}
\hline Instance & $\begin{array}{c}\text { MBA* } \\
\text { (4 threads, 3600s) }\end{array}$ & DPA* \\
\hline B5 & 88590815 & $72155615(2.06 \mathrm{~s})$ \\
X8 & 24875331 & $22265601(59.12 \mathrm{~s})$ \\
\hline
\end{tabular}

Table 3

DPA* vs MBA*

without the symmetry breaking strategy) showing that integrating state-space reductions can greatly benefit to anytime tree search algorithms and probably even to constructive meta-heuristics. Finally, as expected, the waste (lower bound) guide provides the worst results among the 3 considered guides (16\% more waste than the waste percentage guide and $20 \%$ more waste than the waste percentage / average size guide and only 5 best solutions on 50 instances). However, the waste percentage guide and the waste percentage / average size guide provided similar results (with a slight advantage on the latest as it produces $5 \%$ less waste and finds one best solution more). These results are interesting as they show that both guides are complementary. Indeed, they produce 21 (resp. 22) best solutions where they are the only one to obtain them. Thus it is worth using both.

\subsection{DPA*}

Table 3 shows the benefits of using DPA* on instances with only two precedence chains. There is one such instance in each dataset, but the one in dataset $\mathrm{A}$ is trivial to solve, therefore we only consider instances B5 and X8. Unlike $\mathrm{MBA}^{*}, \mathrm{DPA}^{*}$ is not anytime and terminates long before the 3600 seconds time limit. Furthermore, the solutions it returns are significantly better and are even the best-known solutions for both instances.

\subsection{Final results}

Table 4 sums up the challenge final results. Computational experiments have been performed on a computer with an Intel Core i7-4790 CPU @ $3.60 \mathrm{GHz} \times 8$ processor with 31.3 Go of RAM. This configuration is similar to the one of the challenge. Since the challenge, a few adjustments have been made. Therefore, the results presented here slightly differ from the results obtained during the final phase. Compared to the challenge version, the current version performs better: the total waste on dataset $\mathrm{B}$ and $\mathrm{X}$ decreases from $493,600,549$ for the challenge version to $469,910,749$ for the current one. Columns Final phase best 180s and Final phase best 3600 s contain the best solutions found during the final phase. Results annotated with a star indicate that this solution was found by our algorithm during the final phase of the challenge. The Best known column contains the best solution up to our knowledge. They may have been found during the development of the algorithm, with execution times exceeding 3600 seconds or by other teams. Finally, even if it is not indicated in the table, on most of the instances, if the algorithm is run longer, for example, 2 hours, the solution will still be improved.

\section{Conclusion and perspectives}

In this article, we presented a new anytime tree search algorithm called MBA* for the 2018 ROADEF/EURO challenge glass cutting problem. It performs successive iterations, restarting when its heuristic search tree exploration is completed. During the first iterations, it performs aggressive prunings and behaves like a greedy algorithm. As iterations go, the algorithm performs fewer heuristic prunings, and thus gets access to better solutions (at the cost of an increase of the computation time of each iteration). If enough time and memory are available, the algorithm ends up performing an iteration with no heuristic pruning, finding the best solution regarding the branching scheme.

We proposed two guides (waste percentage, and waste percentage / average item size). These guides can find significantly better solutions than using a lower bound (the waste), which is what is usually used in branch and bounds. We also presented a symmetry breaking strategy and showed that it significantly improves the quality of the solutions returned by the algorithm.

Also, we designed another algorithm, DPA*, for instances with only two precedence chains. This algorithm returns the best-knowns on these instances within short times.

This result shows that anytime tree search algorithms from the AI planning community, and branch and bounds from the Operations Research community can benefit from each other, leading to algorithms competitive with classical meta-heuristics. We believe that the representation of anytime tree search algorithms in the Operations Research literature does not reflect the benefits of applying such methods on complex optimization problems. To the best of our knowledge, many of them remain unexplored such as Beam Stack Search (Zhou and Hansen, 2005), SMA* (Russell, 1992) or Anytime Column Search (Cohen et al., 2018).

Motivated by the success of MBA* on this glass cutting application, fontan2020 already adapted it for classical guillotine cutting problems from the literature and showed that even on more fundamental problems, it is still competitive with the other dedicated algorithms from the literature.

\section{References}

Akeb, H., Hifi, M., M'Hallah, R., 2009. A beam search algorithm for the circular packing problem. Computers \& Operations Research 36, 15131528.

Baldi, M.M., Crainic, T.G., Perboli, G., Tadei, R., 2014. Branch-and-price and beam search algorithms for the variable cost and size bin packing problem with optional items. Annals of Operations Research 222, 125141.

Bennell, J.A., Song, X., 2010. A beam search implementation for the irregular shape packing problem. Journal of Heuristics 16, 167-188.

Bortfeldt, A., Jungmann, S., 2012. A tree search algorithm for solving the multi-dimensional strip packing problem with guillotine cutting constraint. Annals of Operations Research 196, 53-71. URL: https: //doi.org/10.1007/s10479-012-1084-7, doi:10.1007/s10479-012-1084-7.

Cohen, L., Greco, M., Ma, H., Hernández, C., Felner, A., Kumar, T.S., Koenig, S., 2018. Anytime focal search with applications., in: IJCAI, pp. 1434-1441. 
Fontan, F., 2019. Theoretical and practical contributions to star observation scheduling problems. Ph.D. thesis. URL: http://www. theses. fr/s162194. publication Title: http://www.theses.fr.

Lodi, A., Monaci, M., Pietrobuoni, E., 2017. Partial enumeration algorithms for Two-Dimensional Bin Packing Problem with guillotine constraints. Discrete Applied Mathematics 217, 40-47. URL: http:// www. sciencedirect.com/science/article/pii/S0166218X15004734, doi:10. 1016/j. dam. 2015.09.012.

Ow, P.S., Morton, T.E., 1988. Filtered beam search in scheduling. The International Journal Of Production Research 26, 35-62.

Russell, S., 1992. Efficient memory-bounded search methods. ECAI-1992, Vienna, Austria .

Wei, L., Tian, T., Zhu, W., Lim, A., 2014. A block-based layer building approach for the $2 \mathrm{D}$ guillotine strip packing problem. European Journal of Operational Research 239, 58-69. URL: http:// www. sciencedirect.com/science/article/pii/S0377221714003464, doi:10. 1016/j. ejor. 2014.04.020.

Zhang, W., 1998. Complete anytime beam search, in: AAAI/IAAI, pp. 425-430.

Zhou, R., Hansen, E.A., 2005. Beam-stack search: Integrating backtracking with beam search., in: ICAPS, pp. 90-98. 


\begin{tabular}{|c|c|c|c|c|c|c|}
\hline Instance & Comments & $\begin{array}{c}\text { Final phase } \\
\text { best } 180 \mathrm{~s}\end{array}$ & $\begin{array}{c}\mathrm{MBA}^{*} / \mathrm{DPA}^{*} \\
180 \mathrm{~s}\end{array}$ & $\begin{array}{l}\text { Final phase } \\
\text { best } 3600 \text { s }\end{array}$ & $\begin{array}{c}\mathrm{MBA}^{*} / \mathrm{DPA}^{*} \\
3600 \mathrm{~s}\end{array}$ & Best known \\
\hline $\mathrm{A} 1$ & Trivial & - & 425486 & - & 425486 & 425486 \\
\hline $\mathrm{A} 2$ & No prec & - & 9506669 & - & 4383509 & 4383509 \\
\hline A3 & & - & 2651880 & - & 2651880 & 2651880 \\
\hline A4 & & - & 3024240 & - & 2924730 & 2924730 \\
\hline A5 & & - & 2924730 & - & 3283653 & 3017223 \\
\hline A6 & & - & 3389640 & - & 3225930 & 3188646 \\
\hline $\mathrm{A} 7$ & & - & 4703760 & - & 4334610 & 3920520 \\
\hline A8 & & - & 9691844 & - & 8378954 & 8378954 \\
\hline A9 & & - & 2664276 & - & 2664276 & 2664276 \\
\hline A10 & & - & 4084381 & - & 4084381 & 4084381 \\
\hline A11 & & - & 4660669 & - & 4622149 & 4358929 \\
\hline $\mathrm{A} 12$ & & - & 2056504 & - & 1879954 & 1879954 \\
\hline A13 & & - & 10226883 & - & 9440433 & 9331293 \\
\hline A14 & & - & 11686638 & - & 10383378 & 10383378 \\
\hline A15 & & - & 12918611 & - & 11108171 & 10828901 \\
\hline A16 & Trivial & - & 3380333 & - & 3380333 & 3380333 \\
\hline A17 & 2 chains & - & 3617251 & - & 3617251 & 3617251 \\
\hline A18 & & - & 5596728 & - & 4983618 & 4983618 \\
\hline A19 & & - & 3654374 & - & 3323744 & 3323744 \\
\hline A20 & Trivial & - & 1467925 & - & 1467925 & 1467925 \\
\hline B1 & No prec & 3232698 & 3765558 & *2 661318 & 3136398 & 2661318 \\
\hline B2 & & *15 635435 & 14312915 & *13 674125 & 13398065 & 11931095 \\
\hline B3 & & 20540813 & 19786463 & 18191093 & 17093273 & 15786803 \\
\hline B4 & & *8 269045 & 8323615 & *8 269045 & 7973725 & 7315675 \\
\hline B5 & 2 chains & 72155615 & 72155615 & 72155615 & 72155615 & 72155615 \\
\hline B6 & & *12 116527 & 12488887 & *11 195257 & 11089327 & 10800427 \\
\hline B7 & No prec & 9601299 & 9177579 & *8 355819 & 7678509 & 6628839 \\
\hline B8 & & *17 865559 & 17152939 & 16067959 & 15840049 & 14398759 \\
\hline B9 & & 18502147 & 19969117 & 17484577 & 17474947 & 16495897 \\
\hline B10 & & 26012183 & 26904563 & *21951533 & 23065403 & 21951533 \\
\hline B11 & & 25251890 & 27312710 & 22584380 & 23820230 & 20626280 \\
\hline B12 & & *15 868657 & 13734007 & *13 958707 & 13120897 & 12514207 \\
\hline B13 & & *28 349055 & 27360375 & *24 471375 & 23078235 & 22657725 \\
\hline B14 & & *9 346480 & 9442780 & *8 656330 & 8377060 & 8023960 \\
\hline B15 & & *27 794441 & 24568391 & *24 517031 & 23088581 & 22619921 \\
\hline $\mathrm{X} 1$ & & *15 508097 & 15302657 & *14 127797 & 14127797 & 13720127 \\
\hline $\mathrm{X} 2$ & No prec & 6034937 & 6083087 & *5 434667 & 4879337 & 4795877 \\
\hline X3 & & *8 285206 & 7649626 & *7 473076 & 7180966 & 6837496 \\
\hline$X 4$ & & 12182072 & 15488372 & 11405252 & 13366562 & 11405252 \\
\hline$\times 5$ & & 5081297 & 4988207 & 4712147 & 4715357 & 4522757 \\
\hline $\mathrm{X} 6$ & & 12565673 & 11031293 & *10 363613 & 9496913 & 9365303 \\
\hline$\times 7$ & & *22 443360 & 22876710 & 21127260 & 21191460 & 20568720 \\
\hline $\mathrm{X} 8$ & 2 chains & *24 788661 & 22265601 & *24 788661 & 22265601 & 22265601 \\
\hline$\times 9$ & & *22 251225 & 22312215 & 20167935 & 20479305 & 20039535 \\
\hline X10 & & *20 110472 & 18778322 & *17 824952 & 17186162 & 16865162 \\
\hline X11 & & *13 489692 & 12802752 & *12 417552 & 11676042 & 11011572 \\
\hline X12 & & *11963845 & 12358675 & *10 583545 & 10503295 & 10246495 \\
\hline X13 & & 15950172 & 14345172 & *13 533042 & 13125372 & 12130272 \\
\hline X14 & & *8 889542 & 8591012 & *8 013212 & 7644062 & 7422572 \\
\hline X15 & & 13990194 & 13710924 & 11682204 & 11682204 & 10882914 \\
\hline
\end{tabular}

Table 4

Computational experiments comparing the proposed approach compared to other contestants 